\title{
Conservative surgery in multiple cusp involvement in tricuspid valve endocarditis
}

\author{
J R Anderson, P Scott, R U Nair
}

\begin{abstract}
Tricuspid and mitral valve endocarditis caused by Staphylococcus epidermidis in a 57 year old previously healthy man with no history of drug abuse presented as bi-ventricular failure and multiple episodes of pulmonary emboli. He was treated for four weeks with intravenous antibiotics and had serial echocardiographic assessment of the vegetation on the tricuspid valve. This was followed by mitral valve replacement, local excision of vegetation from all the three cusps of the tricuspid valve, and autologous pericardial reconstruction of these cusps with functional assessment by perioperative transoesophageal echocardiography. Postoperative cardiac function was excellent and serial echocardiographic assessment confirmed satisfactory tricuspid valve function.

This is believed to be the first recorded case in which autologous pericardial repair was used to reconstruct all the three cusps in a tricuspid valve after excision of vegetations.
\end{abstract}

Bacterial endocarditis of the tricuspid valve accounts for only 5-10\% of all cases of infective endocarditis. ${ }^{1}$ The response to medical treatment is usually good but surgery is indicated when the condition is complicated by repeated pulmonary emboli or if the size of the vegetations increases or remains more than $1 \mathrm{~cm}$ on echocardiography. ${ }^{2}$

\section{Case report}

A 57 year old previously healthy man was admitted on 16 May 1990 with rigors, lassitude, and weight loss. He had had an acute upper respiratory tract infection in December 1989 and remained well until February 1990. Since then he had become unwell and had lost $13 \mathrm{~kg}$. One month before admission he had been prescribed amoxycillin for a throat infection. There was no history of rheumatic fever or intravenous drug abuse.

At admission he was pyrexial $\left(38.8^{\circ} \mathrm{C}\right)$ with a tender nodule on the pulp of his right middle finger. Auscultation revealed a grade III pansystolic murmur at the left sternal edge.
A precordial echocardiogram showed thickened mitral and tricuspid valve leaflets. This was confirmed by transoesophageal echocardiography which also showed vegetations $>1 \mathrm{~cm}$ across on the septal and anterior tricuspid valve cusps. There was mild tricuspid and mitral reflux but left ventricular function was good.

Infective endocarditis was diagnosed and treatment with intravenous antibiotics started. Staphylococcus epidermidis was isolated from the blood but his pyrexia continued despite prolonged medical treatment.

At operation there was a large vegetation on each tricuspid valve cusp. Each vegetation was excised and the cusp was repaired with an autologous pericardial patch. A flexible Duran ring was inserted for additional support and perioperative transoesophageal echocardiography showed no regurgitation.

A large vegetation on the mitral valve was excised and repaired by direct suture with shortening of an elongated chordae. Unfortunately the valve was not competent and had to be replaced with a Medtronic (size 23) prosthesis. The cardiopulmonary bypass was discontinued easily.

Postoperatively, he made an excellent recovery with resolution of all symptoms. An echocardiogram obtained before discharge showed normal prosthetic mitral valve function with only mild residual tricuspid incompetence. Ten weeks after operation he was very well and no tricuspid regurgitation was seen on the echocardiogram.

\section{Discussion}

When surgery is indicated for endocarditis of the tricuspid valve, the options are (a) debridement with valve preservation, (b) valvectomy with prosthetic replacement, and (c) valvectomy without prosthetic replacement.

The mortality from tricuspid valve surgery is high but this may be owing to associated aortic and mitral valve disease and late presentation for surgery. ${ }^{3}$ Earlier intervention before cardiac decompensation occurs may improve the results of surgery.

Valvectomy without prosthetic replacement is associated with troublesome postoperative right heart failure in some patients. ${ }^{1}$ However, since this treatment for persistent right sided 
endocarditis was reported, ${ }^{4}$ more and more cases have been described where it was possible to preserve the tricuspid valve by careful removal of the vegetations..$^{5-7}$ The use of autologous pericardium for tricuspid valve repair has been described before but was used only if two minor leaflets or one major leaflet were involved. ${ }^{8}$

Our case was complicated by the associated endocarditis of the mitral valve where it was not possible to preserve the valve because of coexisting degenerative changes. Apart from this, the tricuspid valve also had vegetations in all three cusps and removal of these with part of the valve cusps resulted in three significant defects. Repair of these defects with autologous pericardium enabled us to maintain good apposition of the valve cusps. This was further reinforced with the insertion of a Duran ring.

In a large series of cases of tricuspid valve surgery, there was no difference in mortality between valve repair and valve replacement. ${ }^{3}$ It is, however, our view that conservative surgery of the atrioventricular valve should be attempted when practical and possible. This case illustrates the feasibility of conserving the tricuspid valve despite the presence of vegetations on all the leaflets.

The excellent postoperative valve function after this repair is very encouraging but more cases are needed to establish that this should be attempted every time this clinical situation occurs.

1 Chan P, Ogilby JD, Segal B. Tricuspid valve endocarditis Am Heart $J 1989 ; 117: 1140-6$

2 Buda AJ, Zotz RJ, Le Mire MS, Bach DS. Prognostic significance of vegetations detected by two dimensional echocardiography in infective endocarditis. Am Heart J 1986;112:1291-6.

3 McGrath LB, Gonzales-Lavin L, Bailey BM, Grunkemeier GL, Fernandez JF, Laub GW. Tricuspid valve operations GL, Fernandez JF, Laub GW. Tricuspid valve operations

in 530 patients. J Thorac Cardiovasc Surg 1990;99:124-33.
4 Arbulu A, Thoms MW, Wilson RF. Valvulectomy without prosthetic replacement, a lifesaving operation for tricuspid pseudomonas endocarditis. $J$ Thorac Cardiovasc Surg 1972;64:103-7.

5 Jagger JD, McGaughan BC, Pawsey CGK. Tricuspid valve endocarditis cured by excision of a single vegetation. $A m$ Heart $J$ 1986;112:626-7.

6 Chandraratna PA, Reagan RB, Imaizui T, Langevin E, Elkins RC. Infective endocarditis cured by resection of a tricuspid valve vegetation. Ann Intern Med 1978;89: 517-8.

7 Tanaka M, Abe T, Hosokawa S, Suengaga Y, Hikosaka $H$. Tricuspid valve candida endocarditis cured by valvesparing debridement. Ann Thorac Surg 1989;48:857-8.

8 Yee ES, Ullyott DJ. Reparative approach for right sided endocarditis. J Thorac Cardiovasc Surg 1988;96:133-40.

\section{Comment}

There are good reasons for Anderson et al to be proud of their technical achievement in repairing all three cusps of a tricuspid valve damaged by endocarditis. None the less, the question remains as to whether for the next patient they encounter this provides a better prospect than replacing the valve. Their view "that conservative surgery of the atrioventricular valve(s) should be attempted when practical and possible" reflects the current tide of opinion. However, in this instance, the attempt at repairing the mitral valve was abandoned because the surgeons regarded the attempted repair as unsatisfactory. The success they achieved vindicates their intraoperative decisions. The case emphasises that the excellent results reported for mitral valve repair include a selection process at this stage, and if bad intraoperative results had been accepted by the pioneers of mitral valve conservation it is likely that conservative operations on the mitral valve would have fallen into disrepute. In the case of the mitral valve there is a growing body of evidence to suggest that repair gives superior short and long term results for the patient. The ideal policy is much harder to establish in tricuspid valve operations because they are so relatively infrequent. ${ }^{1}$ McGrath et al in a series of tricuspid valve operations in 530 cases were unable to demonstrate benefit for a conservative policy over the results achieved with valve replacement. ${ }^{2}$ A recent report, also from Leeds, reports excellent long term results with a tissue valve tricuspid valve replacement in a series of 73 patients followed for, on average, nearly 10 years. ${ }^{3}$ The evidence suggests that, although Anderson, Scott, and Nair achieved an excellent result by conserving the valve, a strategy aimed at replacing the valve with a reliable prosthesis might have done just as well. On the evidence available, an elaborate attempt to conserve a tricuspid valve in a patient with endocarditis who has to have mitral valve replacement to save his life seems hard to justify.

\section{St George's Hospital, \\ Blackshaw Road,}

London SW17 OQT

TOM TREASURE

1 Surgery for tricuspid regurgitation [editorial]. Lancet 1988;ii:1061-2.

2 McGrath LB, Gonzales-Lavin L, Bailey BM, Grunkemeier GL, Fernandez JF, Laub GW. Tricuspid valve operations in 530 patients. J Thorac Cardiovasc Surg 1990;99:124-33.

3 Eng J, Ravichandran PS, Kay PH, Murday AJ. Long-term results of Ionescu-Shiley valve in tricuspid position. Ann Thorac Surg 1990;51:200-3. 